\title{
Analysis the Services of Multicast and Broadcast in Heterogeneous Network using QualNet6.1
}

\author{
Neelam Yadav \\ M.Tech Scholor \\ Department of \\ Electronics \& \\ Communication \\ SSET, SHIATS \\ Allahabad, India
}

\author{
Rajeev Paulus \\ Professor \\ Department of \\ Electronics\& \\ Communication \\ SSET, SHIATS \\ Allahabad, India
}

\author{
A.K Jaiswal \\ HOD \\ Department of \\ Electronics\& \\ Communication \\ SSET, SHIATS \\ Allahabad, India
}

\author{
Aditi Agrawal \\ Assistant professor \\ Department of \\ Electronics\& \\ Communication \\ SSET, SHIATS \\ Allahabad, India
}

\begin{abstract}
Mobile devices can exist in many forms. IEEE 802.11 and IEEE 802.16 create a heterogeneous network. Heterogeneity implies that some devices are more powerful than others, and some can be servers while others can only be clients. Typical IEEE $802.11 \mathrm{~b} / \mathrm{g} / \mathrm{a}$ is considered up to $54 \mathrm{Mbit} / \mathrm{s}$.WiMax IEEE [802.16] has the specification of Medium Access Control (MAC) also Physical (PHY) layers. The results analyze the validity of broadcast and multicast in heterogeneous networks. The basic idea is to enhance the performance of multicasting and broadcasting in heterogeneous network. The evaluation is done through extensive simulations conducted in QualNet 6.1 simulator.
\end{abstract}

\section{Keywords}

Heterogeneous networks, IEEE 802.16e, mobile WiMax, IEEE802.11 Wi-Fi, Multicast and broadcast service, QualNet 6.1

\section{INTRODUCTION}

Now a day, the trend of communication system is migrating from wired networking to wireless networking. Efficiency of energy in wireless networks is mostly impacted by the power consumption which has become most one of the interesting areas of research. Energy consumption is also becoming a crucial factor for wireless networks [1]-[2]. A heterogeneous network involves a mix of radio technologies and cell types working together seamlessly to deliver the additional capacity, coverage and speed needed to secure excellent user experience. A heterogeneous network is a network connecting computers and other devices with different operating system and/or protocols. For example, local area networks (LANs) that connect Microsoft windows and Linux based personal computers with Apple Macintosh computers are heterogeneous. A wireless network which provides a service through wireless LAN and is able to maintain the service when switching to a cellular network is called a wireless heterogeneous network The main driver for the heterogeneous network vision- that $\mathrm{Wi}-\mathrm{Fi}$, along with Heterogeneous networks accomplish this by combining radio-access technologies (RATs) to boost peak capacity at hotspots and improve performance at cell edges and in buildings. This requires cost-efficient easy-to-install radio solutions that complement the existing macro network in high-traffic areas. Mobile operators have three ways to enhance network capacity and coverage to meet growing mobile-broadband demand [4]

-Enhance the macro network with additional licensed spectrum, more antennas and better processing capabilities, possibly through carrier aggregation.

-Density the macro network. Such site densification offers an attractive solution by keeping the total number of sites relatively low, while network performance is less sensitive to traffic location.

-Add capacity through small-cell deployment using lowpower.

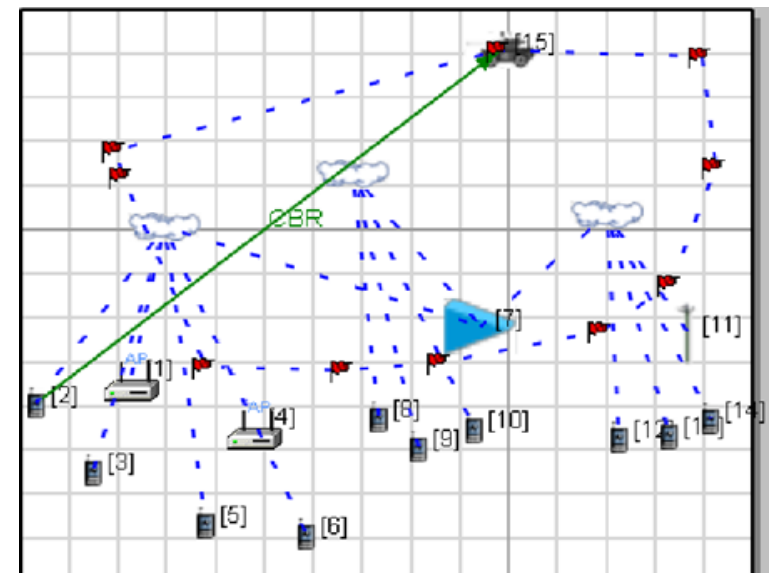

Fig.1. Heterogeneous Network with mobility

WiMaX stands for worldwide interoperability for microwave access ,it is a wireless communication network based on the IEEE802.16 standard [5]WiMax (worldwide interoperability for microwaves access) is wireless communications standard designed to provide 30-40 Mbps data rate, with the 2011 update providing up to $1 \mathrm{Gbps}$ for fixed stations. WiMax is standard based technology enabling the delivery of last mile wireless broadband access as an alternative to cable and DSL. $\mathrm{Wi}-\mathrm{Fi}$ is local area wireless technology that allows an electronic device to exchange data or connect to the internet using $2.4 \mathrm{GHz}$ UHF and $5 \mathrm{GHz}$ SHF radio waves. It can be less secure than wired connections (such as Ethernet) because an intruder does not need a physical connection. Web pages that uses SSL are secure but unencrypted internet access can easily be detected by intruders. Because of this, Wi-Fi has adopted 
various encryption technologies. The early encryption WEP, proved easy to break. QualNet which is a commercial spin-off from the Glomo sim simulator based on $\mathrm{C}++$ programming. All protocol are implemented in a series of $\mathrm{C}++$ files, and called by the simulation kernel. One of advantages of QualNet is that it is more scalable. During the simulation time, it enables the user can see the all signals being transmitted and received at each node, which assists in the understanding of what is happening in a freshly manner. QualNet simulator used the three main programs such as the analyzer and the packet tracer. When simulator runs the given simulation, analyzer displays the simulation results and packet tracer aids to realize the path of a packet through the network. There is also a protocol developer in the GUI, however, it is much more powerful to develop protocols from $\mathrm{C}++$ coding [9]. QualNet allows investigation and evaluation of fixed and mobile WiMax devices, applications and networks. WiMax channel model of QualNet includes co-channel interference, urban path loss, fading, shadowing and mobility effects. Through detailed models, unrivaled speed and scalability, QualNet [11]

\subsection{Broadcast and Multicast Services}

Multicast and Broadcast Services (MBS) are supported by Heterogeneous network. Network-wide broadcasting and multicasting are two important routing schemes used in group communications. In network-wide broadcasting, generated packets at the source node are distributed to all nodes in the network, while multicasting delivers the packets only to a subset of the nodes by creating and maintaining a data dissemination structure. Due to the overhead in multicasting, in certain situations, it is more efficient to use network-wide broadcasting instead of multicasting, even when the data is destined to a subset of the nodes.[6] and satisfy the following requirements.

-Flexible allocation of radio resources

-Low MS power consumption

-Support of data-casting in addition to audio and video streams

-Low channel switching time.

The trend of communication system has been changing from wired network to wireless network. Heterogeneous network has been developed to overcome the limitations of the existing wireless communication system such as bandwidth problem. The main concept of Heterogeneous network is to enhance the performance of broadcast and multicast where mobility also take place.[8] Mobile WiMax offers high speed Internet service whereas $\mathrm{Wi}-\mathrm{Fi}$ provides other application which provides various information and multimedia data with high data rate on broadband regardless of place and time. The Mobile WiMax Air Interface adopts Orthogonal Frequency Division Multiple Access (OFDMA)

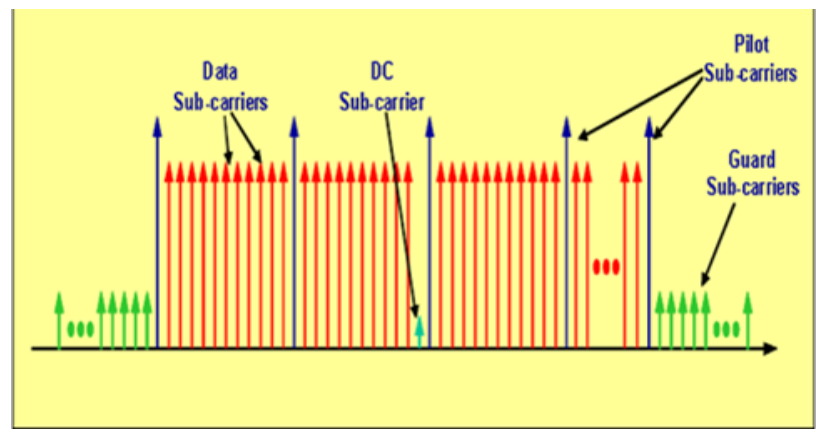

Fig.2. OFDMA Subcarrier Structure
The OFDMA symbol structure consists of three types of subcarriers as shown in fig.

- Data sub-carriers for data transmission

- Pilot sub-carriers for estimation and synchronization purposes

- Null sub-carriers for no transmission; used for guard bands and DC carriers.

Active (data and pilot) sub-carriers are grouped into subsets of sub-carriers called sub channels. The WiMax OFDMA PHY supports sub-channelization in both DL and UL.The minimum frequency-time resource unit of sub-channelization is one slot, which is equal to 48 data tones (sub-carriers).

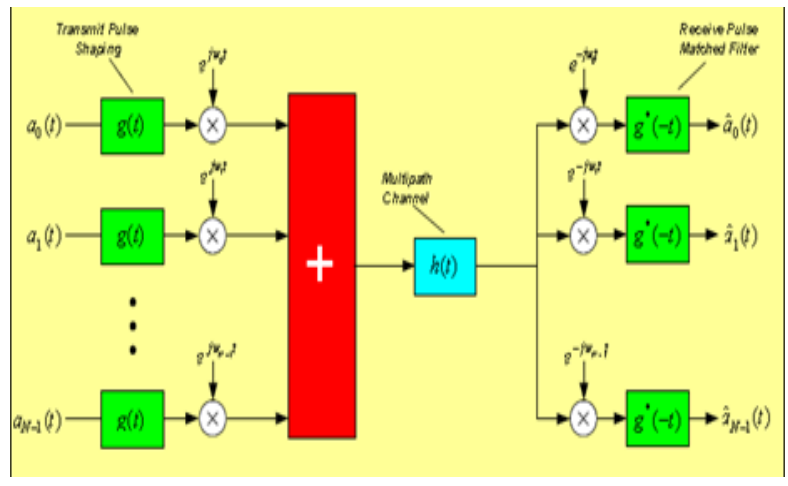

Fig.3. Basic Architecture of OFDM

\subsection{Heterogeneous Network}

A heterogeneous network is understood as a combination of various network technologies (wired / wireless) put together for efficient data connectivity across the network. Heterogeneous network environments illustrate the integration and coexistence of a variety of wireless access technologies with different protocol stacks and supporting a number of applications and services with different QoS requirements to be provisioned to network resources with different degree of multi-mode capabilities to access the available networks [13]. To make use of multiple broad ba The requirement of Heterogeneous Networks arises from the need of an efficient coexistence of latest and legacy technologies. For example, the new emerging technologies like HSDPA \& WiMax will have to coexist with legacy technologies like GSM, GPRS. A heterogeneous network also includes telecommunication networks viz. 3GPP-standardised UTRAN and GERAN along with non-cellular access networks like WLAN 802.11.nd transport technologies and to support generalized mobility. 


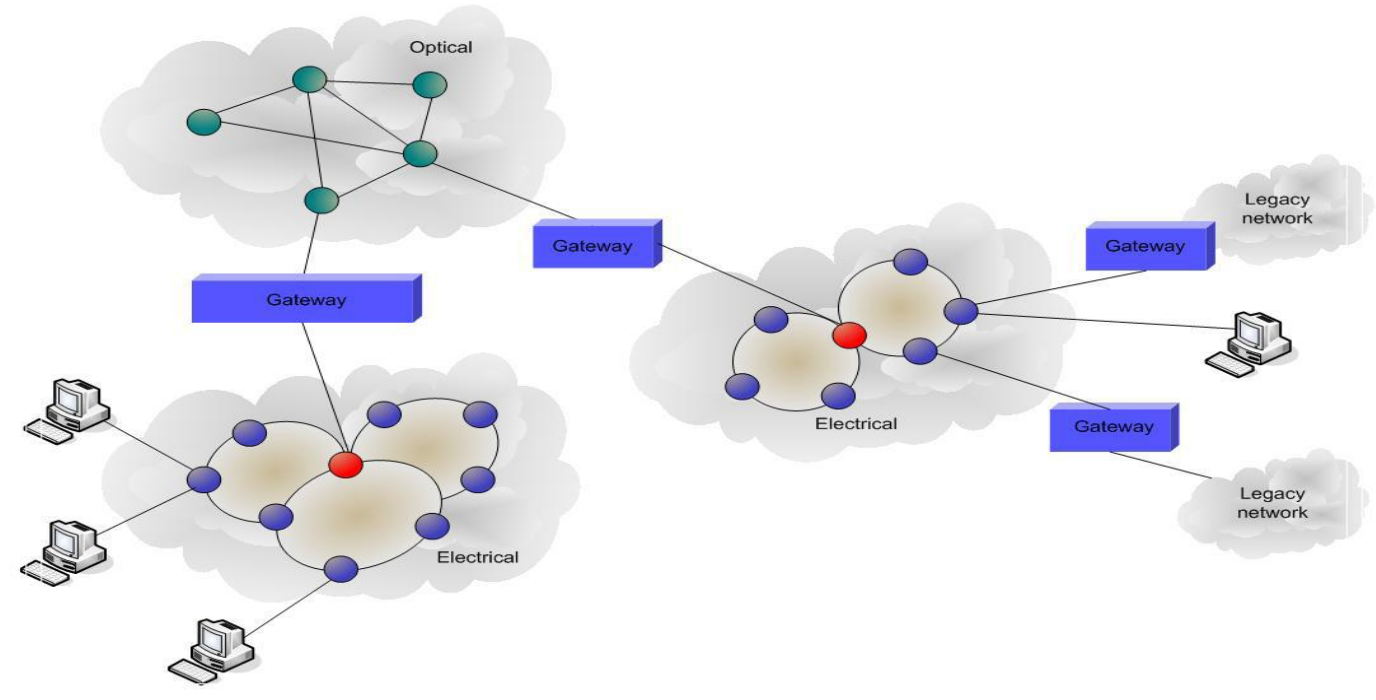

Fig 4. Structure of Heterogeneous Network

\section{ANALYSIS OF SIMULATION IN QUALNET}

The paper design a simulation model for multicast and broadcast service in heterogeneous network which has been designed by QualNet 6.1. This scenario is divided in two zones consists of access point base station and rest are mobile station with two router and one content provider i.e Hub.MBS zone 1 consists of three access point which are connected to mobile station and MBS zone 2 consists of two base stations which is connected to six mobile stations which is shows in Fig.3. Each base station is connected with a wireless subnet and communication takes place from zone1 to zone 2 by using a inter connector node called content provider. For transmission purpose CBR and MCBR applications are used. Here CBR application is used for unicast of one packet per second with a simulation time of $530 \mathrm{sec}$. The zone covers $1500 \mathrm{~m} \times 1500 \mathrm{~m}$ area for transmission purpose. MCBR is connected to specified mobile stations which are given as MS6, MS9, MS12, and MS18 with CBR (constant bit rate) and MCBR is used for multicast constant bit rate with the packet size of 505 bytes and an packet interval of $1 \mathrm{sec}$. The packets are started at $1 \mathrm{sec}$ and end time of simulation is530sec.

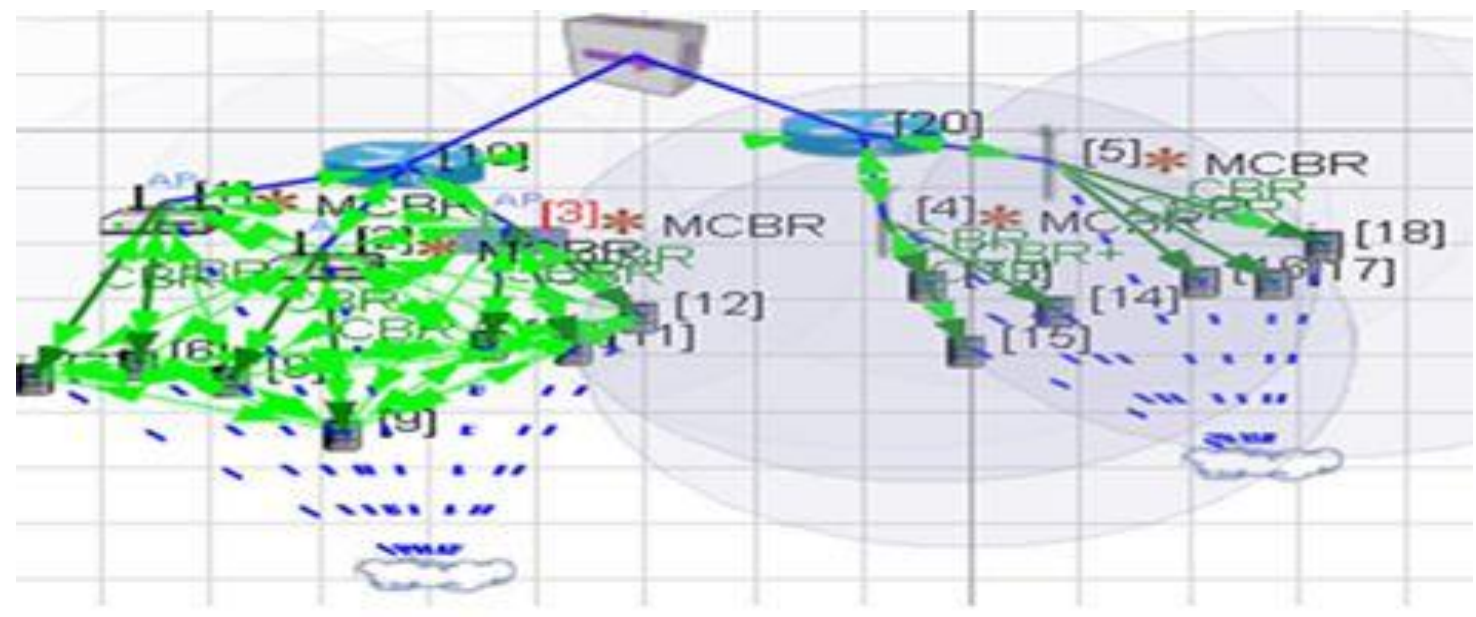

Fig.5.Simulation model of Heterogeneous MBS in QUALNET 
Table 1. Simulation Parameters:

\begin{tabular}{|l|l|}
\hline Parameters & Values \\
\hline Channel Frequency & $2.4 \mathrm{GHZ}, 2.42 \mathrm{GHZ}$ \\
\hline Channel bandwidth & $10 \mathrm{Mhz}$ \\
\hline Simulation area & $1500 * 1500$ \\
\hline Simulation time & $530 \mathrm{sec}$ \\
\hline Number of node & 20 \\
\hline Packet size & 505 \\
\hline Users in the zone 1 & MS6-MS12 \\
\hline User in the zone 2 & MS13-MS18 \\
\hline Node placement & Random \\
\hline Traffic type & CBR and MCBR \\
\hline FFT size & 2048 \\
\hline Interval & 1 sec \\
\hline Simulation start time & 1 sec \\
\hline Multicast protocol & DVMRP(IGMP) \\
\hline Antenna type & Omnidirectional \\
\hline Radio propagation method & Two ray ground \\
\hline Multicast address & $224.0 .1 .0,224.0 .1 .1$ \\
\hline End time & 530 sec \\
\hline
\end{tabular}

\section{RESULTS}

This paper work has been carried out by simulation through QualNet6.1 which defines the parameters for the performance evaluation of Broadcast and Multicast in a heterogeneous network under different propogation model and varying packet rate i.e packet per second. With the help of QualNet Simulator, the simulation result has been analyzed for Received Throughput, Average end to end delay and Average Jitter values for given Heterogeneous network. The graphical results are given as follows:

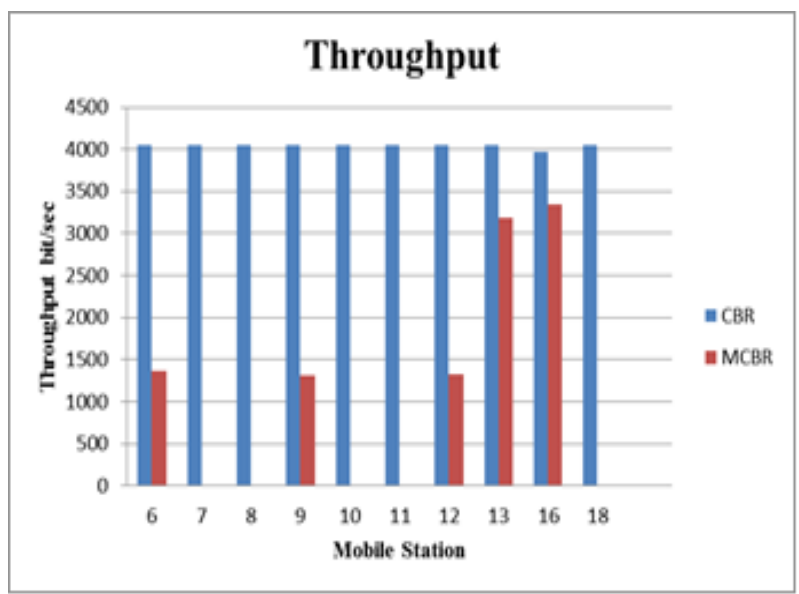

Throughput is the rate of successful message delivery over a communication channel. Throughput in the heterogeneous network is shown from the fig. 6 MCBR is connected at the nodes [6], [9], [12], [13], [16] and [18]. The received throughput is analysed by varying CBR \& MCBR.

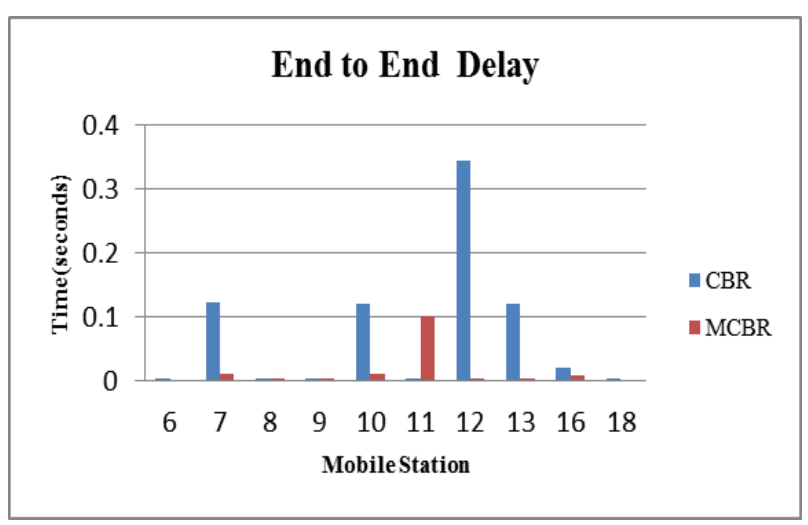

Fig.7. Average End-to-End Delay (seconds)

From the graphs we see that the average packets delay increased with number of nodes while routing protocols try to find valid route to the destination. The average time taken by a data packet to arrive in the destination is called end-to-end delay. End to End delay is very less in case of Wi-Fi but at the side of WiMax it will gives delay as shown on node [13], [16] sand [18].

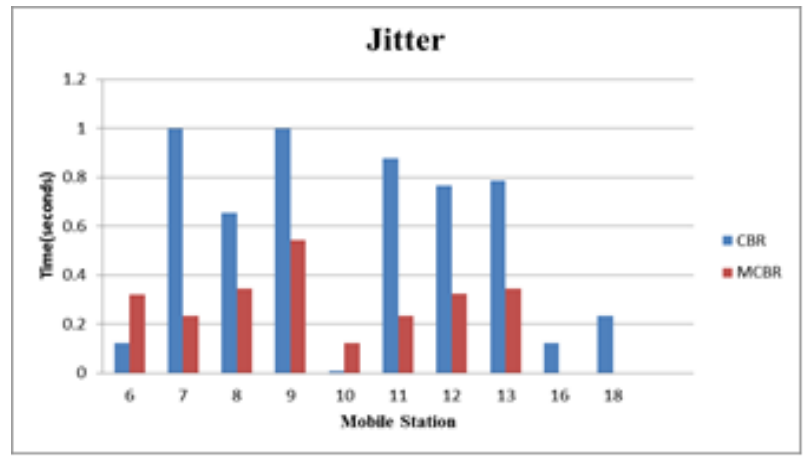

Fig.8. Average Jitter (seconds)

Average jitter is the variation in the time between packet arriving caused by network congestion, timing drifting, or route change. Average jitter should be minimum for a routing protocol to perform better. The level of jitter is less in both either it is CBR or MCBR hence the performance of Heterogeneous network is improved here the minimum jitter will observe at node [7] and maximum on node [9] which is using MCBR

\section{CONCLUSION}

The performance of multicasting and broadcasting services in Heterogeneous network has been analyzed in this paper by using QUALNET Simulator 6.1. Heterogeneous network gives better result. The result from the scenario shows several performance measures such as received throughput, end to end delay and average jitter. We can change the result by varying the parameters such as cyclic prefix factor, FFT size and packet size. We can also use multiple antennas to increase data rate transmission for IPTV service. We also checked it for the overall energy consumption.

Fig.6 Unicast Received Throughput (bits/second) 


\section{ACKNOWLEDGMENT}

I wish to express my gratitude to those who in some or the other way has helped me in accomplishing the challenging paper on the topic "Analysis the services of Multicast and Broadcast in Heterogeneous Network using QualNet6.1 Keeping aside the arrogance of enthusiastic spirit of human nature all my heartfelt thanks to the God Almighty who bestowed upon me zeal as a result of which this arduous task is completed for the growth and betterment of humanity and mankind. I would like to express my deep sense of gratitude to Prof. Rajeev Paulus, for his appropriate guidance. I express my deep sense of gratitude to my parents for their constant support and motivation without them this would not had been reality. I thank my friends for their encouragement in this regard and look forward in completing and achieving new possibilities in this study. Also, I would like to thank all the staff of the department for their constant support and help all the way through.

\section{REFERENCES}

[1] Amit B. Kalyani (2012) "Heterogeneous Network Framework Architecture - A Survey" IRACST International Journal of Computer Science and Information Technology \& Security (IJCSITS), ISSN: 2249-9555 .Vol. 2, No.4, August 2012

[2] Bora Karaoglu, Wendi Heinzelman (2000) “ Multicasting vs. Broadcasting: What are the Trade-offs?" Department of Electrical and Computer Engineering University of Rochester.

[3] C.F. Ball, E. Humburg, K. Ivanov, F. Treml (2004) "Comparison of IEEE802.16 WiMax Scenarios with Fixed and Mobile Subscribers in Tight Reuse" Siemens AG, Communications Mobile Networks Munich, Germany.

[4] Chi Hyun Cho, Kyung Tae Kim, and Hee Yong Weng,(2008)" Mobile Multi-hop Relay System using AMC for Multicast Broadcast Service over Mobile WiMAX", Korea.

[5] Haitao Wu, Shiduan Cheng,Yong Peng, Keping Long, Jian Ma IEEE(2002) "IEEE 802.11 Distributed Coordination Function(DCF): Analysis and Enhancement" National Key Lab of Switching Technology and Telecommunication Networks, Beijing 100876.
[6] IEEE802.11.(1999) "Wireless LAN Medium Access Control (MAC) and Physical Layer (PHY) Specifications" First Edition.

[7] Jong Min Lee, Hyo-Jin Park, Seong Gon Choi,and Jun Kyun Choi, Senior Member, IEEE (2005)“Adaptive Hybrid Transmission Mechanism for On-Demand Mobile IPTV Over WiMAX

[8] Khishigjargal Gonchigsumlaa, Young-Il Kim (2011) "The Simulation Model of Multicast and Broadcast Service in the Mobile WiMAX for QualNet" IPTV Structure Research Team, ETRI, University of Science and Technology, Daejeon, Korea.R.China

[9] LapKongLaw, V.Krishnamurthy, and Michalis Faloutsos (2011)"Understanding and Exploiting the Tradeoffs between Broadcasting and Multicasting in Mobile Ad Hoc Networks" USA

[10] Manish Gupta, Jiawei Han(2001) "Heterogeneous Network-Based Trust Analysis: A Survey" SIGKDD Explorations Volume 13, Issue 1 Page 54.

[11] Mahdi Aiash, Glenford Mapp, Aboubaker Lasebae, Jonathan Loo,(2009) " A Survey of Potential Architectures for Communication in Heterogeneous Networks". UK

[12] Prof. K.H. Wandra, Dr. Ketan Kotecha (2010) "Various effect of modulation schemes on Wi-Fi Network using Qualnet Simulator" IEEE 2nd International Confe -rence on Education Technology and Computer (ICETC).

[13] Steve Vandris, Kai-Uwe Killiches "Power Optimization in Wireless Heterogeneous Networks" LSI White paper.

[14] Suman Banerjee, Archan Misra, Jihwang Yeo, Ashok Agrawala (2007) "Energy-Efficient Broadcast and Multicast Trees for Reliable Wireless Communication" Dept. of Computer Science, University of Maryland, College Park, MD, USA.

[15] Yongguang Zhang,Son K. Dao,Harrick Vin,(2000) "Heterogeneous Networking: A New Survivability Paradigm" Conference, USA.

[16] Z.J. Haas and M. R. Pearlman.(2000) "The zone routing protocol: A hybrid framework for routing in ad hoc networks" in Ad Hoc Networks, C. E.

[17] QUALNET Advanced Wireless Library (WIMAX) QUALNET, 2008 